\title{
Advancements in preclinical screening methods for artemisinins during pregnancy
}

\author{
G Donegan", C A Forrester, S A Ward \\ From Parasite to Prevention: Advances in the understanding of malaria \\ Edinburgh, UK. 20-22 October 2010
}

\section{Background}

An unprecedented effort from public and private international health agencies has been made to address the urgent issue of malaria in pregnancy.

The selection of antimalarial drug therapy during pregnancy currently depends upon the severity of the disease, gestational age (stage of fetal development) and patterns of drug resistance in the area. To combat multi-drug resistance the WHO recommends include the use of Artemisinin-based combination therapies.

To assist in policy formulation of arteminisin drug combination therapy during pregnancy, artemisinin-derivatives are currently undergoing preÂAclinical risk:benefit assessments [1]. Recent animal trials have indicated that arteminisins during pregnancy result in fetal morbidity, low birthweight, cardiac and skeletal birth defects [2].

Currently validated preclinical teratogenicity/embryolethality drug screen $\hat{A} \neg$ ing includes whole embryo culture (WEC), Micromass (MM) limb bud and embryonic stem cell (ESC) culture together with in vivo studies to assess embryonic development [3,4].

However while in vivo screening assays to gestational day (GD) 13+ enable unambiguous assessment of artemisinin-associated embryotoxicity, standard in vitro 48hr WEC studies from 9-11 GD end before the teratogenic effects on vulnerable organ systems impacted by the drug can be evaluated. To address this problem, the WEC experiment is extended to 72 hours: after vascular and limb bud development.

\section{Materials and methods}

For in vivo and in vitro studies timed-mated Sprague Dawley female rats were administered with artesunate at 9 and 10GD (organogenesis). Validated total morphological scoring systems are used to quantify development of the heart, brain, mandibular and maxillary processes, skeletal formation and otic and optic development [2].

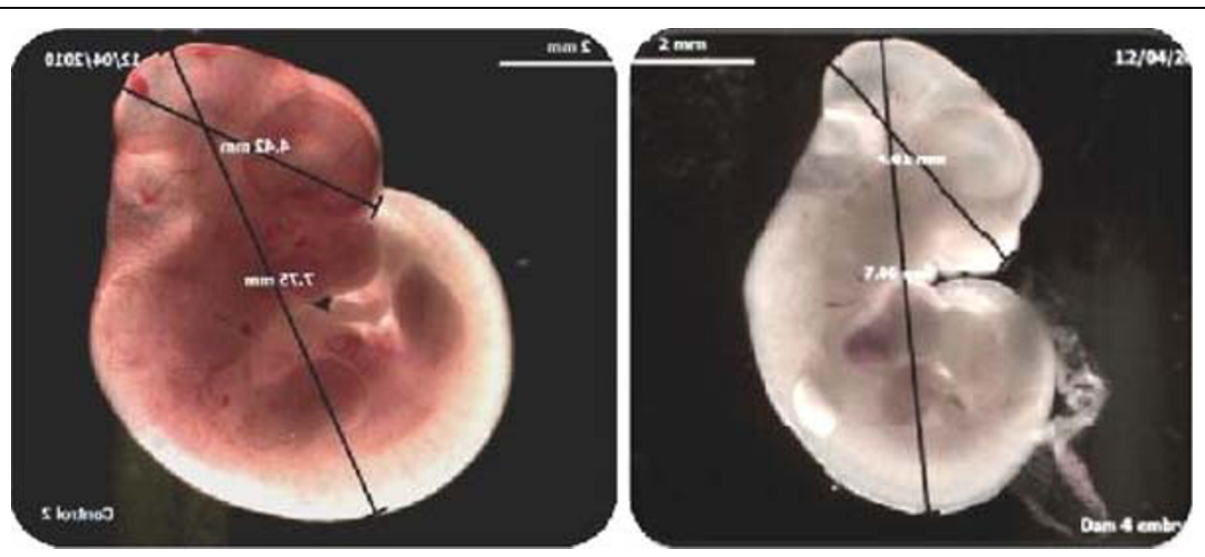

Figure 1 In vivo Control (L) and Artesunate-treated (17mg/kg) gestation day 12 .

Molecular \& Biochemical Parasitology Group, Liverpool School of Tropical Medicine, Liverpool, L3 5QA, UK 


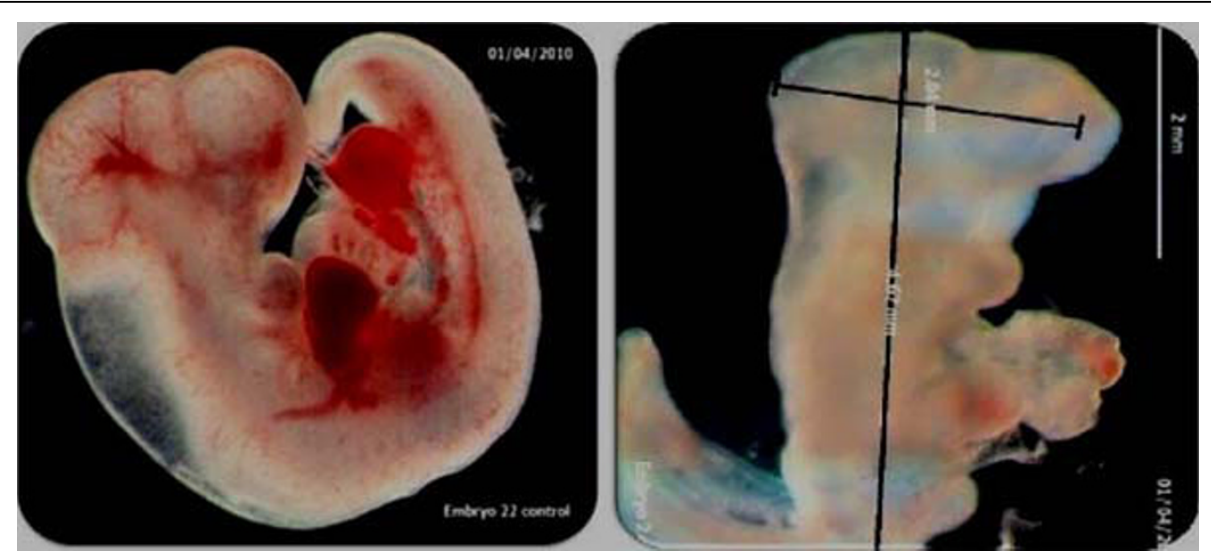

Figure 2 In vitro Control (L) and Artesunate-treated $(0.5 \mu \mathrm{g} / \mathrm{mL})$ gestation day $12 \mathrm{WECs}$ at $72 \mathrm{hr}$.

Embryonic blood was collected for haematological analysis and de novo blood vessel formation of blood vessels in the yolk-sac was also assessed.

\section{Results}

Extended (72 hours from gestational day 9-12) whole embryo culture times clearly showed artesunate (an artemisinin-derivative) to have dose-dependent effects with total morphological scores increasing as dose was reduced. Unequivocal effects were upon embryonic vascularisation, fore-limb bud development, morphological scores and 50\% reduced fetal weight were clearly demonstrated that would not have been seen after 48 hours of culture.

Analogous effects were confirmed in the in vivo experiments at $17 \mathrm{mg} / \mathrm{kg}$ (Fig 1). The earliest effects were observed in yolk-sac size and vascularisation showing $50 \%$ reduction in fetal weight, $20 \%$ reduction in head size and severely reduced vascular development at 13 GD. There was also a $40 \%$ reduction in numbers of nucleated erythroid progenitors. Figure 2.

\section{Conclusions}

These studies seek improve harmonization between the current in vitro developmental toxicity assays and in vivo assays for use in preclinical drug testing. The results indicate that for compounds with teratogenic potential specifically on skeletal and cardiac development, $72 \mathrm{hr}$ whole embryo culture assay is a prerequisite for valid in vitro assessment and to enable more relevant equivalence with in vivo data.

Published: 20 October 2010

\section{References}

1. Report of two joint informal consultations convened in 2006 by: The Special Programme for Research and Training in Tropical Diseases (TDR) sponsored by UNICEF/UNDP/World bank/WHO and The global Malaria
Programme of the World Health Organization. Assessment of the safety of artemisinin compounds in pregnancy [http://apps.who.int/tdr/publications/ tdr-research-publications/artemisinin-compounds-pregnancy/pdf/ artemisinin-compounds-pregnancy.pdf].

2. Longo, et al: In vivo and in vitro investigations of the effects of the antimalarial drug dihydroartemisinin (DHA) on rat embryos. Reprod Toxicol 2006, 22:797-810.

3. Detection of toxicity to reproduction for medicinal products including toxicity to male fertility. (ICH S5A) European Medicines Agency [http://www. ema.europa.eu/pdfs/human/ich/038695en.pdf].

4. Piersma $\mathrm{AH}$, et al: Validation of the postimplantation rat whole embryo culture test in the international ECVAM validation study on three in vitro embryotoxicity tests. Altern Lab Anim 2004, 32:275-307.

5. Brown NA, Fabro S: The value of animal teratogenicity testing for predicting human risk. Clin Obstet Gynecol 1983, 26(2):467-77.

doi:10.1186/1475-2875-9-S2-P8

Cite this article as: Donegan et al:: Advancements in preclinical screening methods for artemisinins during pregnancy. Malaria Journal 2010 9(Suppl 2):P8.

\section{Submit your next manuscript to BioMed Central and take full advantage of:}

- Convenient online submission

- Thorough peer review

- No space constraints or color figure charges

- Immediate publication on acceptance

- Inclusion in PubMed, CAS, Scopus and Google Scholar

- Research which is freely available for redistribution
Biomed Central 